\title{
Vertically polarizing undulator with dynamic compensation of magnetic forces
}

\author{
N. Strelnikov, ${ }^{1,2}$ I. Vasserman, ${ }^{1}$ J. Xu, ${ }^{1,}{ }^{*}$ D. Jensen, ${ }^{1}$ O. Schmidt, ${ }^{1}$ E. Trakhtenberg, ${ }^{1}$ K. Suthar, ${ }^{1}$ \\ E. R. Moog, ${ }^{1}$ G. Pile, ${ }^{1}$ and E. Gluskin ${ }^{1}$ \\ ${ }^{1}$ Advanced Photon Source, Argonne National Laboratory, Argonne, Illinois 60439, USA \\ ${ }^{2}$ Budker Institute of Nuclear Physics, Novosibirsk 630090, Russia
}

(Received 6 October 2016; published 20 January 2017)

\begin{abstract}
As part of the R\&D program of the LCLS-II project, a novel 3.4-meter-long undulator prototype with horizontal magnetic field and dynamic force compensation has recently been developed at the Advanced Photon Source (APS). Previous steps in this development were the shorter 0.8-meter-long and 2.8-meterlong prototypes. Extensive mechanical and magnetic testing were carried out for each prototype, and each prototype was magnetically tuned using magnetic shims. The resulting performance of the 3.4-meter-long undulator prototype meets all requirements for the LCLS-II insertion device, including limits on the field integrals, phase errors, higher-order magnetic moments, and electron-beam trajectory for all operational gaps, as well as the reproducibility and accuracy of the gap settings.
\end{abstract}

DOI: 10.1103/PhysRevAccelBeams.20.010701

\section{INTRODUCTION}

Undulators are critical components of synchrotron radiation (SR) sources and free electron lasers (FEL). In order to deliver high-quality radiation, undulators have to meet challenging tolerances in their magnetic and mechanical performance.

Most SR sources and FELs utilize undulator insertion devices (IDs) with a vertically oriented magnetic field [1,2]. This direction is preferred because the electron beam cross section in the storage ring is much wider horizontally than it is tall, so that the smallest gap (and strongest magnetic field) can be obtained when the gap is vertical. Although the electron beam cross section in an FEL is circular, ID designers have not yet taken real advantage of the opportunity this presents to rotate the undulator. This status quo could soon change because of recent advancements in the design of ultra-small-emittance storage rings. Such machines promise to operate with nearly circular e-beams and on-axis injection. Therefore, the development of novel planar IDs with horizontal magnetic fields becomes a practical matter not only for FELs but also for storage rings.

There are at least two major advantages to rotating the ID geometry by $90^{\circ}$. One is that it rotates the polarization plane of the undulator radiation to vertical so that horizontal reflections preserve the most photon flux. Monochromators and experimental setups transform to "gravity-neutral" systems [3] with horizontal deflections, in many cases

\footnotetext{
*x@anl.gov

Published by the American Physical Society under the terms of the Creative Commons Attribution 3.0 License. Further distribution of this work must maintain attribution to the author $(s)$ and the published article's title, journal citation, and DOI.
}

significantly simplifying their construction and operation. The second advantage is also related to the "gravityneutral" design but now pertains to the ID mechanical system itself. When such a design is combined with a distributed magnetic-force compensation system, the ID gap drive mechanism can become quite compact without sacrificing stringent requirements on the accuracy and reproducibility of ID gap control.

Currently, all FELs around the world use a traditional approach in the design of ID gap drive mechanisms, whether the ID is out of vacuum, in vacuum, APPLE-type, etc. This traditional approach uses very strong, often bulky, strongback beams that are able to withstand tremendous magnetic forces without noticeable distortion, and have very precise mechanical components that permit control of the ID magnetic gap value to $\mu \mathrm{m}$-level precision. In some cases the fabrication of such strongbacks requires unique machine tools that can process several-meter-long beams with a precision of $10-15 \mu \mathrm{m}$. Also, the size of the strongbacks makes these IDs quite space consuming in the tight and valuable volumes of FEL ID tunnels.

Recently, after more than a decade of developments, the European XFEL has successfully constructed several dozen 5-m-long IDs with a very sophisticated ID drive system that meets XFEL specifications [4]. The newly built XFEL in Pohang, South Korea, adopted the European XFEL ID design [5]. The ID team at Lawrence Berkeley Lab that is building a set of IDs for the LCLS-II soft x-ray beam line also follows the XFEL ID design approach [6]. The SACLA XFEL in Japan has followed the design of invacuum IDs developed for the SPring-8 storage ring [7], and the Swiss FEL follows SACLA's footsteps by choosing in-vacuum IDs [8] but with a quite different, complex gap drive system. The FERMI FEL in Trieste, Italy [9] is using 


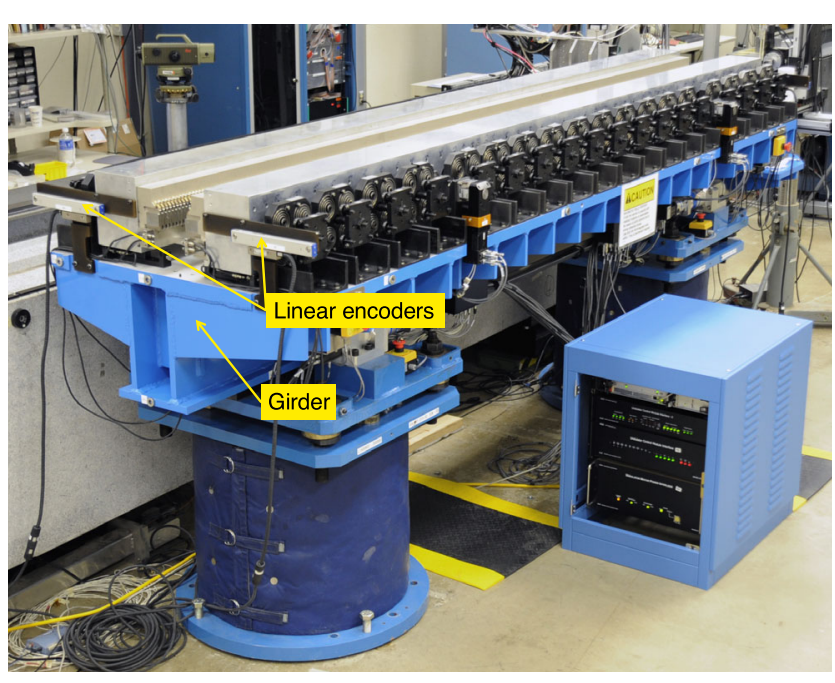

FIG. 1. LCLS-II horizontal gap vertically polarizing undulator (HGVPU).

APPLE-type IDs for its soft x-ray FEL with their own drive mechanism. All of these devices have a vertical main magnetic field and the massive strongbacks that hold the magnetic structures are usually quite tall. None of those devices can be turned into horizontal devices due to their size in the main field plane.

ID designs with different types of magnetic force compensations have been implemented or prototyped at several SR facilities [10-14], but none of them led to a reduction in ID dimensions or noticeable simplification of the ID mechanical system. A novel ID recently developed by the APS for the LCLS-II project uses a magnetic force compensation system [15] based on custom-designed conical springs. Such an approach resulted in a significant reduction of the ID volume, simplification of the strongback design and fabrication, and the reorientation of the $\mathrm{x}$-ray polarization plane into the vertical direction.
The first, short (0.8-m-long) prototype of the ID was designed, built, and tested at the Advanced Photon Source (APS) of Argonne National Laboratory [16]. The ID magnetic forces were compensated by a set of conical springs placed along the ID strongback. The wellcontrolled exponential characteristics of the conical springs permitted a very close fit to the gap-dependent ID magnetic forces. The magnetic measurements of the undulator effective field demonstrated that the magnetic gap within the operating range was controlled accurately and reproducibly within $\pm 1 \mu \mathrm{m}$. Successful tests of this ID prototype led to the design of a 3.4-m-long device with 260 poles per jaw based on the same concept. This longer undulator, shown in Fig. 1, was also successfully built and tested at the APS. It has been included in the baseline of the LCLS-II project for the hard x-ray beam line. The device also represents R\&D efforts by the APS toward APS upgrade project goals. This paper describes the main features and mechanical and magnetic performance of this novel undulator.

\section{GAP DRIVE MECHANISM}

The novel ID gap drive mechanism, shown in Fig. 2, is the main feature of the HGVPU that allows the gap to be controlled with a precision of $0.2 \mu \mathrm{m}$ and the mechanical deformation of the strongbacks to be limited to $10 \mu \mathrm{m}$ over the full range of operational gaps from 7.2 to $20 \mathrm{~mm}$. The strongbacks are made of aluminum because it is nonmagnetic and easily machined. The $\mathrm{x}$-dimension of the strongbacks (the dimension that affects stiffness most strongly) is limited by the maximum allowed footprint of the undulator. The gap drive mechanism includes: a base plate or girder, two linear slides, four drive actuators, linear encoders, and magnetic force compensation units or spring units. The drive actuators can be and are quite small because the strong magnetic forces are compensated by the

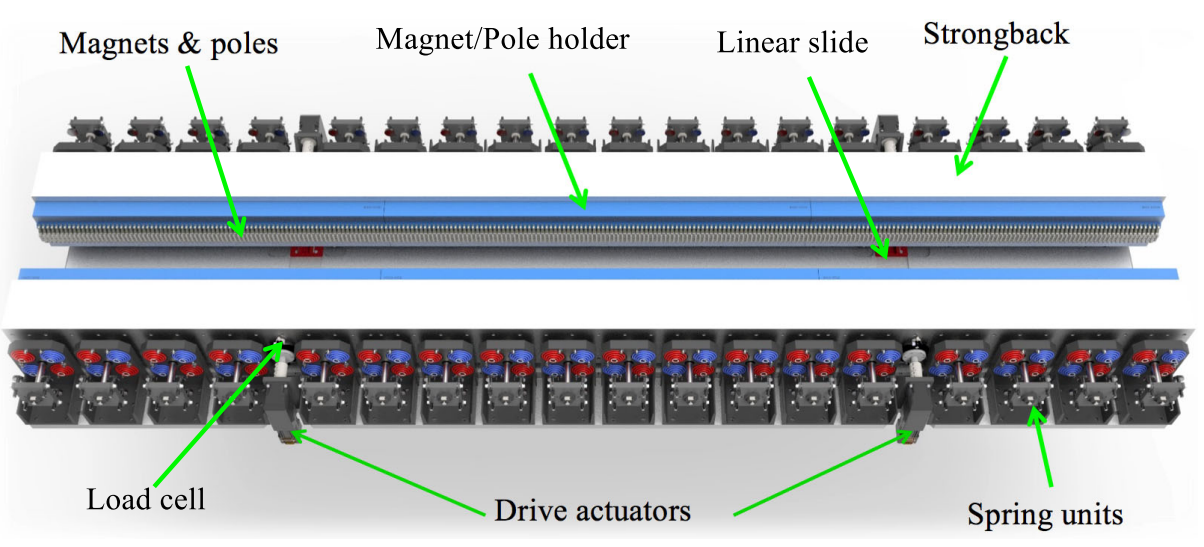

FIG. 2. Design rendering of the HGVPU gap drive mechanism. The linear slides are at the same positions along the undulator as the drive actuators. The blue and red coloring on the springs in the spring units indicates weaker and stronger, respectively. Combining their response curves and adjusting the engagement points using the spring calibration buttons (see Fig. 3) allows a better match to the gap dependence of the magnetic force. 


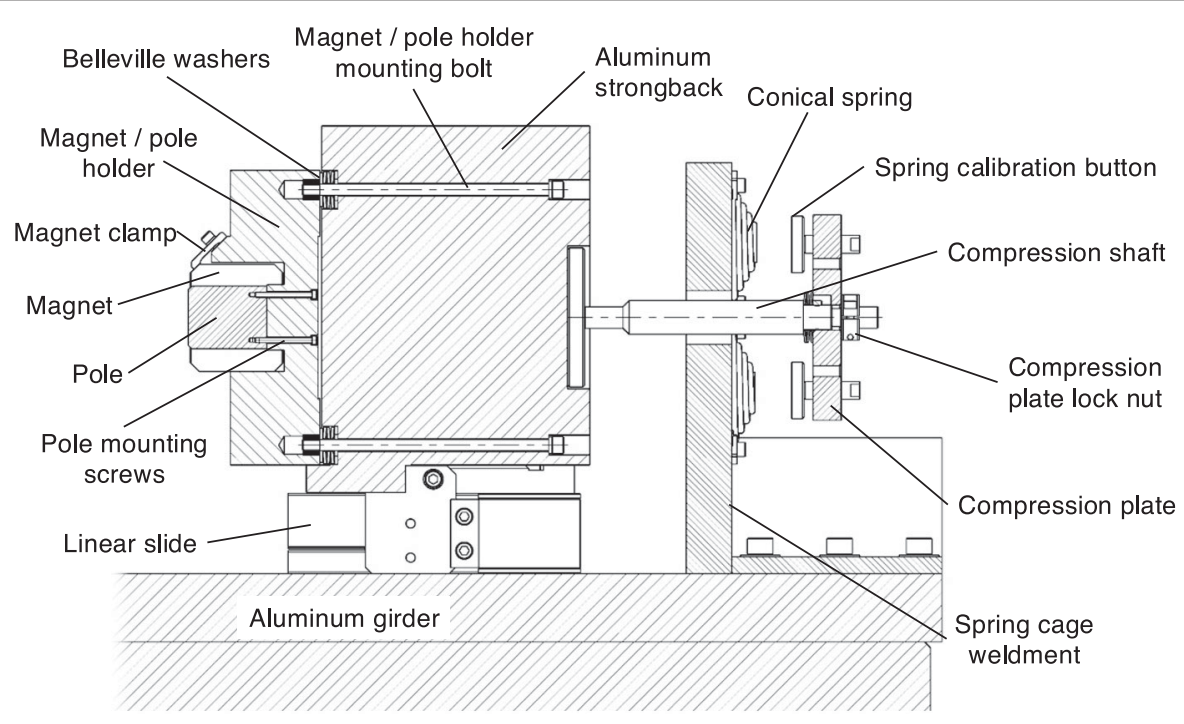

FIG. 3. Cross-sectional view of the undulator strongback, magnet/pole holder, and spring unit.

spring units so little force is needed from the actuators. Details of the spring unit assembly are shown in Fig. 3.

There are 18 spring units for each strongback. Each spring unit consists of four conical compression springs of two types (two of each type), a spring cage weldment bracket that the springs are mounted to and that is rigidly fixed to the ID girder seen in Fig. 1, a compression shaft which is attached to the strongback and being pulled by it, a compression plate, and spring calibration buttons. The compression plate is fixed on the shaft by a lock nut which is used to adjust the position of the compression plate and the resulting spring unit engagement point. Magnets and poles are placed in aluminum "comb" structures, or magnet/pole holders. As shown in Fig. 3, each pole has two threaded holes that are used to bolt it to the holder. Magnets are secured between poles by magnetic forces and safety clamps. Each of the six holders (three per undulator jaw) is attached to the undulator strongback by long bolts. There is a small gap introduced between strongback and holders by inserting a set of Belleville washers. The washers are compressed enough to significantly exceed the maximum magnetic force at any gap so that the position of the magnet/pole mounts relative to the aluminum strongback is determined by the magnet/pole holder mounting bolt. The undulator gap can therefore be adjusted locally by a small amount to aid in mechanical tuning. This tuning is similar to that provided by mechanical shims between the strongback and magnet/pole holder, but much more efficient. Four linear encoders permit the undulator gap to be controlled with an accuracy of less than $0.2 \mu \mathrm{m}$.

\section{MAGNETIC DESIGN}

A design for a 2.6-cm-period undulator should meet the requirements given in Table I. This section describes how those requirements were applied in the design of the magnetic structure. The final undulator parameters are given below in Table II.

The grade of magnet chosen was grade $\mathrm{N} 42 \mathrm{SH}$ from Shin-Etsu. It has a remanent field $\mathrm{B}_{r}$ of $12700-13200 \mathrm{G}$ and an intrinsic coercivity $\mathrm{H}_{\mathrm{cJ}}$ of $>21000 \mathrm{Oe}$; it has been used successfully in several recently built undulators at the Advanced Photon Source.

The choice of recess of the magnet tips relative to the pole tips is a tradeoff: space is needed for shim placement, but it comes at a cost in on-axis field. The usual compromise of $0.5 \mathrm{~mm}$ was chosen. A nominal space of $0.0625 \mathrm{~mm}$ is allowed between magnet and pole to accommodate dimensional tolerances and for ease of assembly.

The OPERA codes [17] were used for the magnetic modeling. Two-dimensional calculations were used to determine the magnet and pole "heights" (in the horizontal direction the gap opens, or $x$ direction), thicknesses (in the beam or $\mathrm{z}$ direction), and the sizes of the chamfers on the $\mathrm{z}$ edges. Choices were made based on the joint goals of maximizing the effective field and minimizing the demagnetizing field in the magnet. The magnet height was tall

TABLE I. Requirements, per LCLSII-1.1-DR-0001-R0.

\begin{tabular}{lc}
\hline \hline Parameter & Requirement \\
\hline Period & $26 \mathrm{~mm}$ \\
Minimum gap & $7.2 \mathrm{~mm}$ \\
$\Delta \mathrm{K}_{\text {eff }} / \mathrm{K}_{\text {eff between undulator segments }}$ & $<2.3 \times 10^{-4}$ \\
Electron beam position relative & $\leq 1 \mathrm{~mm}$ \\
$\quad$ to magnetic center & \\
Tunnel temperature & $20^{\circ} \mathrm{C}$ \\
$\mathrm{B}_{\text {eff }}$ & $>1.005 \mathrm{~T}$ \\
$\mathrm{~K}_{\text {eff }}$ & $>2.44$ \\
Magnet grade $\mathrm{Br}$ & $>1.3 \mathrm{~T}$ \\
Magnet grade $\mathrm{H}_{\mathrm{cJ}}$ & $>21 \mathrm{kOe}$ \\
\hline \hline
\end{tabular}


TABLE II. Design parameters for the 2.6-cm-period undulator. Notation on the chamfers: PM_chzx, PM_chzz are the $\mathrm{x}$ and $\mathrm{z}$ dimensions of the $\mathrm{z}$ chamfer. (Note that $\mathrm{x}$ is horizontal and $\mathrm{y}$ is vertical on the component dimensions when the undulator is in its horizontal-gap configuration.)

\begin{tabular}{lllcc}
\hline \hline & In cm & In mm & $\begin{array}{c}\text { Tolerance } \\
\text { in cm }\end{array}$ & $\begin{array}{c}\text { Tolerance } \\
\text { in mm }\end{array}$ \\
\hline Period & 2.6 & 26 & & \\
Minimum gap & 0.72 & 7.2 & & \\
Air_gap (used & 0.00625 & 0.0625 & & \\
$\quad$ in modeling) & & & & \\
Magnet Y & 6 & 60 & \pm .025 & \pm .25 \\
Magnet X & 5.2 & 52 & \pm .005 & \pm .05 \\
Magnet Z & 0.785 & 7.85 & \pm .005 & \pm .05 \\
PM_chzz & 0.11 & 1.1 & \pm .012 & \pm .12 \\
PM_chzx & 0.05 & 0.5 & \pm .012 & \pm .12 \\
PM_chyy & 0.5 & 5 & \pm .012 & \pm .12 \\
PM_chyx & 0.5 & 5 & \pm .012 & \pm .12 \\
Magnet recess & 0.05 & 0.5 & & \\
Pole Y & 3.495 & 34.95 & \pm .005 & \pm .05 \\
Pole X & 4.3995 & 43.995 & \pm .0005 & \pm .005 \\
Pole Z & 0.5025 & 5.025 & \pm .0025 & \pm .025 \\
Pole_chzz & 0.02 & 0.2 & \pm .005 & \pm .05 \\
Pole_chzx & 0.02 & 0.2 & \pm .005 & \pm .05 \\
Pole_chyy & 0.2 & 2 & \pm .025 & \pm .25 \\
Pole_chyx & 0.2 & 2 & \pm .025 & \pm .25 \\
\hline \hline
\end{tabular}

enough that further height increases only resulted in small gains in field strength, but not so tall as to be mechanically inconvenient.

Three-dimensional modeling was used to set vertical "widths" (in y). The roll-off requirement was determined by applying the field uniformity requirement $\left(\Delta \mathrm{K}_{\text {eff }} / \mathrm{K}_{\mathrm{eff}}<\right.$ 0.0002 ) over the beam position accuracy of $\pm 1 \mathrm{~mm}$. Since the roll-off requirement is harder to meet at larger gaps, the $\mathrm{x}$-ray tuning range $(1.5$ to $5 \mathrm{keV}$ with a $4-\mathrm{GeV}$ electron beam) determined the relevant gap range to be 7.2 to $20 \mathrm{~mm}$. The requirement is met by the $35-\mathrm{mm}$ pole width chosen. The magnet width is wide enough that the return from additional width is diminishing and narrow enough to remain mechanically convenient. Figure 4 shows a sketch of the magnetic design and Table II gives the final design parameters along with the machining tolerances on the magnet and pole dimensions.

\section{STRONGBACK DEFORMATIONS}

One of the most important requirements for an undulator is that of low phase errors [18], and phase errors strongly depend on the gap profile shape [19]. Therefore, strongback distortions, such as "bowing," should be kept to an acceptable level. In the HGVPU, the magnetic attractive force between the jaws is countered by the spring units, but the distributions of those forces along the length of the strongback are different. The magnetic forces are nearly uniformly distributed along the undulator, except at the undulator ends. The spring units, however, generate pointlike forces that sum to the same value as the total magnetic force. For convenience in production, the spring units are identical, so that each one provides the same force (i.e., $1 / 18$ of the total magnetic force) and the same gap dependence of the force. While customizing the spring units for particular locations could reduce the strongback deformation, the mechanical requirements can be readily

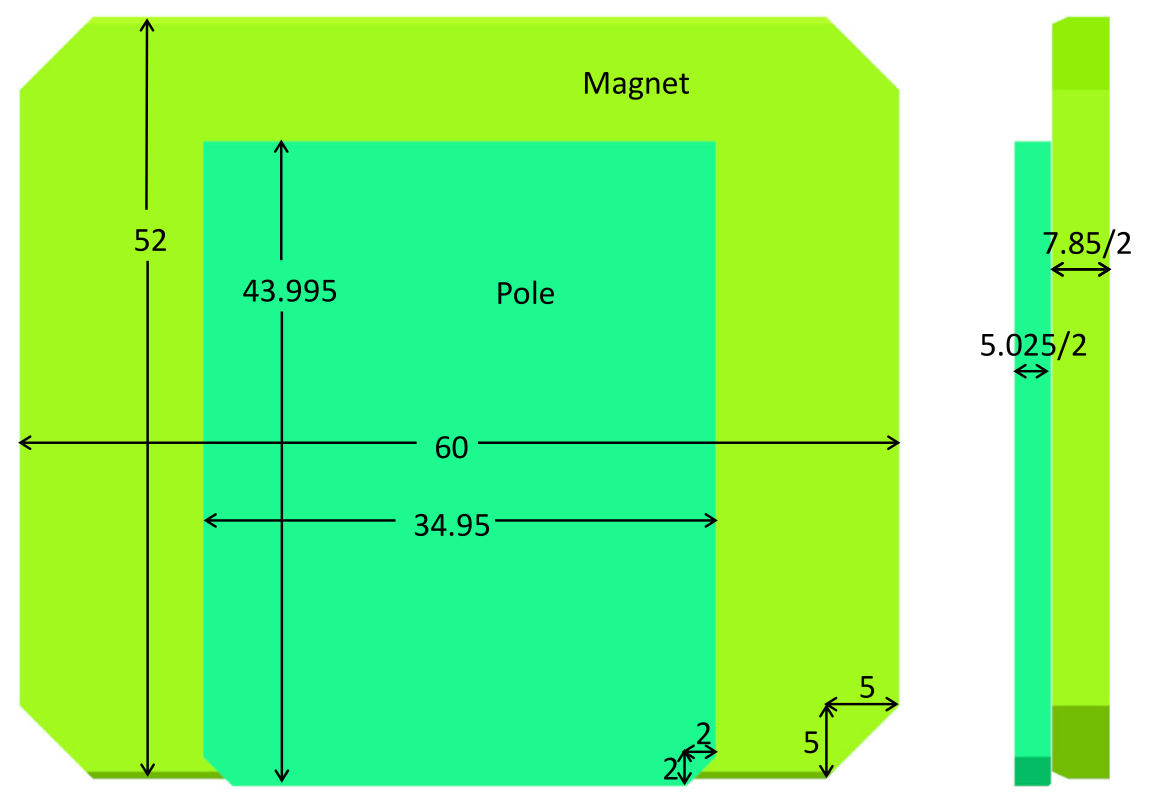

FIG. 4. Sketch of a quarter period of the magnetic design; only half of the thicknesses of the magnet and pole are shown. Dimensions are $\mathrm{mm}$. $\mathrm{Z}$ chamfer dimensions and the magnet recess are given in Table II. 
met without the additional complication of nonuniform spring units, as will be shown below.

Ideally, the force provided by the actuator is near zero. In reality, however, there is some lumpiness in the force curves of the springs despite the sorting of the springs into spring units (see below), so the actuators must exert some gap-dependent force. The $2.8-\mathrm{m}$ prototype showed that, because of this residual force, optimized $\mathrm{z}$ positioning of the actuators is important. Optimizing the placement of the actuators also involves adjustment in the placing of the spring units. Although the spring units are nearly evenly spaced, the even spacing of the units between the actuators may be different from the even spacing of the units between the actuators and the undulator ends.

Optimizing the locations of the drive actuators and the 18 spring units can minimize strongback distortion. Two optimization methods were used: the numerical solution of Eq. (1) and 3D modeling using COMSOL MULTIPHYSICS [20]. The strongback deformation $x(z)$, where $z$ is distance along the length of the undulator and $x$ is distance in the gap-opening direction, follows the equations [21,22]:

$$
\begin{aligned}
& E I \frac{d^{2} x}{d z^{2}}=\int_{0}^{z} \int_{0}^{z^{\prime}} \omega\left(z^{\prime \prime}, x\right) \mathrm{d} z^{\prime \prime} d z^{\prime}+C_{0} z+C, \\
& \omega(z, x)=\omega_{0}(z, x)-\sum_{i} F_{i}(x) \delta\left(z-z_{i}\right),
\end{aligned}
$$

where $E$ is the elasticity modulus of the beam material, $I$ is the strongback moment of inertia, $\omega_{0}(z, x)$ is a gapdependent magnetic load distribution, $F_{i}(x)$ are point forces that include the spring unit forces and the reaction forces of the actuators when there are errors in the spring units, $\delta\left(z-z_{i}\right)$ is a delta function, $z_{i}$ are the locations of the point forces, and $C_{0}$ and $C$ are constants found from boundary conditions.
Magnetic field calculations for the HGVPU, including the nonperiodic fields at the ends of the undulator, were carried out using both the OPERA [17] and RADIA [23,24] codes. The field distribution in the midplane of the undulator was calculated for several gaps between 7.2 and $20 \mathrm{~mm}$. That field was then used to determine the distribution of the force along the strongback $[25,26]$ :

$$
d F=\frac{B_{x}^{2}}{2 \mu_{0}} d y d z
$$

The solution of Eq. (1) for the case of ideal and identical spring units is shown in Fig. 5 for the optimum locations of the actuators (the supports in the simulation model) and spring unit attachment points. The optimum is where the peak-to-peak beam deformation is minimized. Although the spring units are evenly spaced between the actuators and evenly spaced outside the actuators, those spacings are not the same. The magnetic force is weaker at the undulator end due to the reduced-strength end magnets, so a larger spacing between spring units at the ends is appropriate. As can be seen in the figure, this calculation gives a maximum deformation of the strongback of $4.7 \mu \mathrm{m}$ at a gap of $7.2 \mathrm{~mm}$.

The undulator pole heights are required to be within $10 \mu \mathrm{m}$ of the nominal height at the 7.2-mm minimum gap. This is both to ensure small phase errors (see below) and to avoid interference with the (nominally) $7.0-\mathrm{mm}$ vacuum chamber. At larger gaps, interference with the vacuum chamber is not a problem and the phase error requirement can be achieved with a somewhat relaxed gap tolerance. One option for adjusting the pole heights to make the gap uniform is to make the adjustments when the undulator is at minimum gap using the magnet/pole holder mounting bolts (discussed below). When the gap is then opened, the relaxation in the gap uniformity requirement is larger than the small gap-dependent changes in the uniformity. For

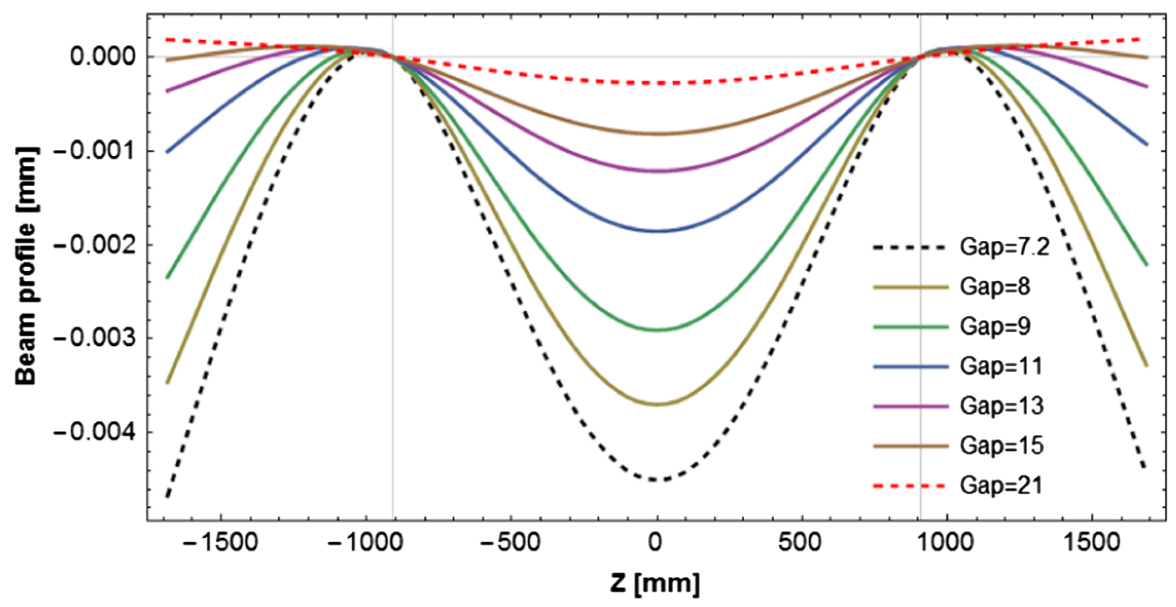

FIG. 5. Analytical calculation of the strongback deformation for optimized actuator locations assuming ideal spring units. The vertical lines mark the actuator locations. 
mass production of the undulators, however, it is preferable to set the pole-tip flatness of one jaw by itself, when the undulator is effectively at open gap. That means that the gap-dependent changes must be less than $10 \mu \mathrm{m}$ so that the uniformity requirement is met at minimum gap. The $4.7-\mu \mathrm{m}$ maximum deformation at $7.2 \mathrm{~mm}$ gap shown in Fig. 5 is small enough to meet the requirement.

The deformation in the strongback was also calculated using the COMSOL code [20]. The locations of the actuators and spring units were the same as in the analytical solution. Results are shown in Fig. 6. In this calculation, the maximum deformation is somewhat smaller: $2.7 \mu \mathrm{m}$ instead of $4.7 \mu \mathrm{m}$ at $7.2 \mathrm{~mm}$ gap. The discrepancy between the analytical and modeled results can be attributed to a difference in the mechanical models: the analytical approach only takes into account the strongback and its stiffness, whereas the finite element model additionally includes the stiffness contributed by the three separate sections of the magnet/pole holder.
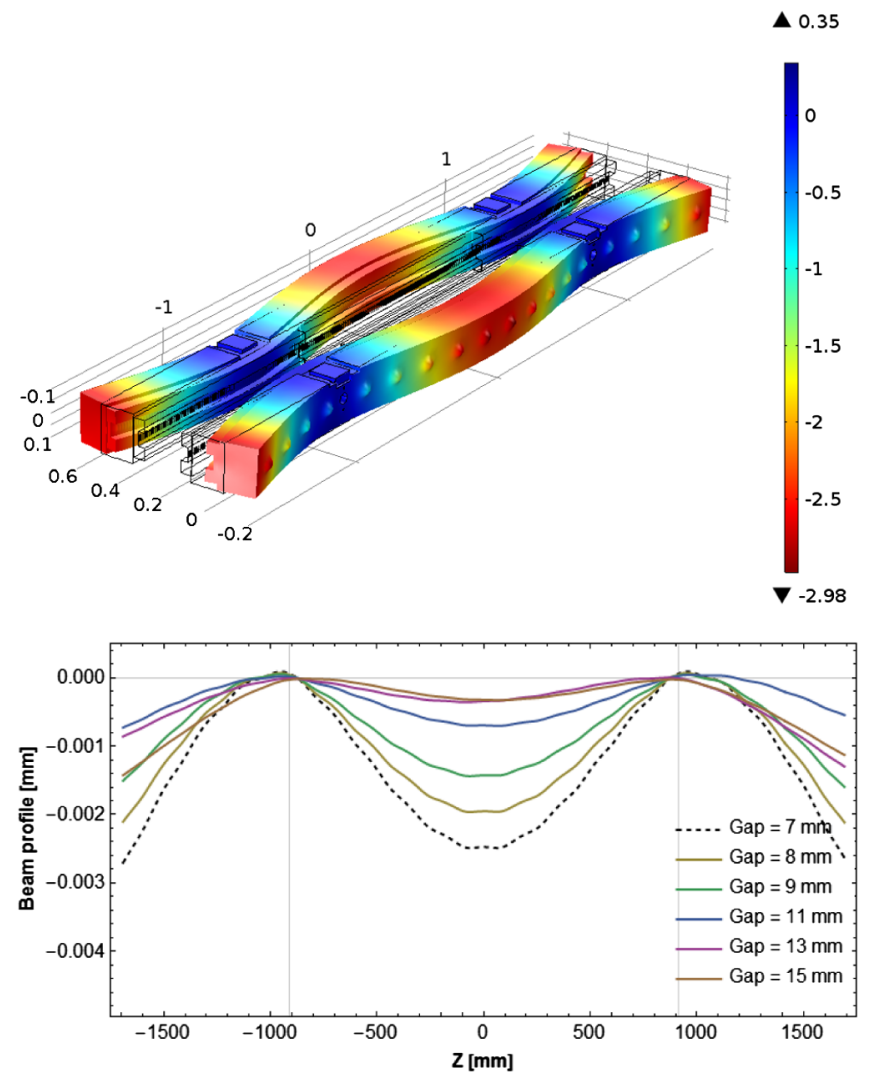

FIG. 6. Simulation results of the strongback deformation, calculated using COMSOL and using actual forces from the spring units. The deflection shown in the upper panel corresponds to a gap of $7.2 \mathrm{~mm}$. Unlike the analytical result of Fig. 5, which included only the stiffness of the strongback, this simulation includes stiffness from the strongback and the sections of magnet/ pole holder. The deformation is therefore somewhat smaller at the same gap.
The shape of the strongback deformation affects the rms phase error caused by a given amplitude of deformation. This was included in the optimization of the locations of actuators and spring units. Phase errors have been calculated for several different shapes of the undulator gap deformation. The results for a simple bowed arc (U shape) and a W shape are shown in Fig. 7 where the beam deformation that results in a $4^{\circ} \mathrm{rms}$ phase error is shown as a function of gap. A W-shaped deformation allows a larger peak-to-peak deformation at almost all gaps; both shapes require that the deformation be below 7 or $8 \mu \mathrm{m}$ at the $7.2 \mathrm{~mm}$ minimum gap. This is smaller than the $10-\mu \mathrm{m}$ requirement stated above for the mechanical straightness. Magnetic tuning after the mechanical alignment is completed will further reduce the rms phase error. As a result, this $7-\mu \mathrm{m}$ acceptable beam deformation would apply when considering reproducibility requirements for the mechanical straightness.

Both systematic and random errors in the spring unit forces have been considered in the analysis of the deformation patterns of the HGVPU gap. The main contribution to the systematic error is spring fading, or a degradation of the spring strength after repeated compression cycles. According to the spring manufacturer, the maximum fading of a 4-spring unit would be $1.5 \mathrm{~kg}$. When the actuator is set for a particular undulator gap and the spring unit strengths have faded, the missing force is taken up by the actuators, resulting in a W-shaped deformation.

Random errors in spring units also affect strongback deformation, so modeling was done to set a tolerance on

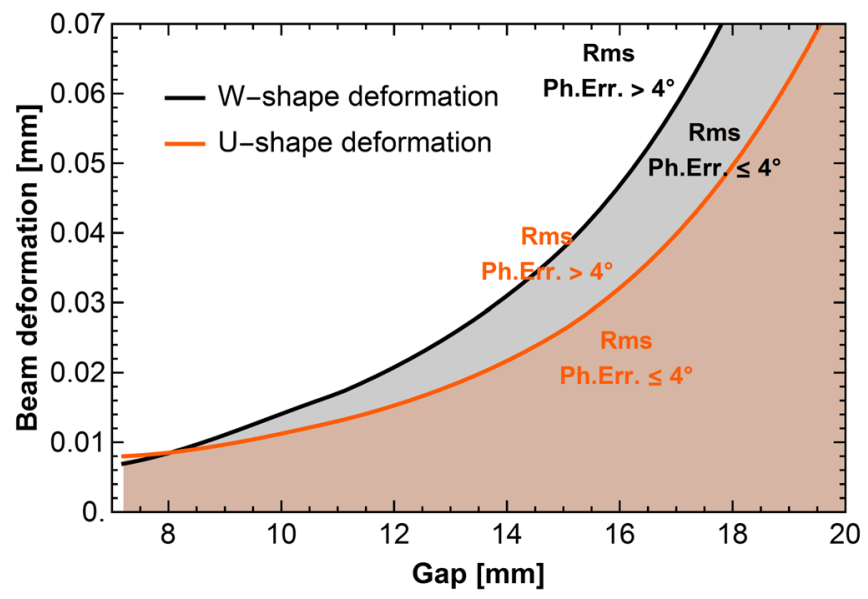

FIG. 7. The strongback deformation (peak to peak) that results in an rms phase error matching the $4^{\circ}$ requirement is shown as a function of gap for two different shapes of deformation. At the minimum gap of $7.2 \mathrm{~mm}$, a deformation that exceeds 7 or $8 \mu \mathrm{m}$ (depending on deformation shape) will result in excessive rms phase error. The deformation can be allowed to be larger at larger gaps. Note that this only includes mechanical shape. On a real undulator, magnetic tuning (see below) will further improve the rms phase error, but the mechanical shape must reproduce to this accuracy. 


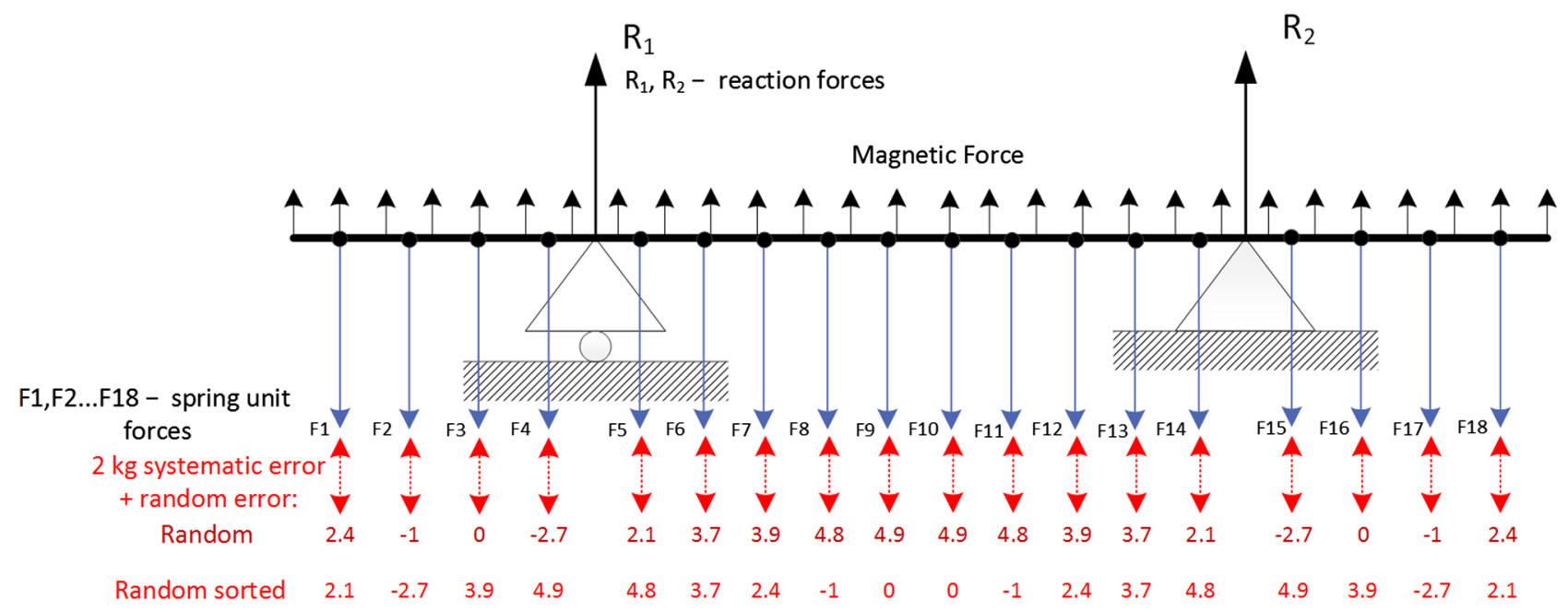

FIG. 8. Schematic of systematic and random errors in spring unit forces (in $\mathrm{kg}$ ). The magnetic force is uniform along the undulator except for the weaker ends (not shown but accounted for in modeling). The spring unit forces are applied at points F1-F18. The uniform spacing between F1-F4 and F15-F18 is slightly larger than the uniform spacing between F5-F14. "Random" shows the random errors between $\pm 5 \mathrm{~kg}$ that were added to the systematic $2 \mathrm{~kg}$ error. The bottom line shows the sorting of the units according to their random errors. The smallest error is in the undulator's middle; the largest errors are close to the actuator since the actuator compensates for any overall force error to hold the desired gap. (The errors shown are symmetric left to right because the model assumed that symmetry.)

those errors. The combined effect of a 2-kg systematic error (from spring fading) and a 5-kg random error was calculated for 200 different sets of random errors. An attempt was also made to choose a worst-possible scenario, but the worst-possible (before sorting) scenarios that were considered turned out to improve slightly more after sorting than the worst of the random error sets. So, the worst of the 200 random cases is shown in Figs. 8 and 9, both before and after sorting of the spring units to minimize beam deformation and phase errors. The $\mathrm{U}$ shape in the beam profile is typical of random errors. In the sorting, the spring units with the largest absolute error were placed closest to the actuators, and the ones with the smallest error were placed closest to the undulator center. As shown in Fig. 9, the total deformation and overall rms phase error can be reduced to meet the requirement by this simple sorting of the spring units.

\section{FORCE-COMPENSATING SPRING UNITS}

The choice of the springs for the spring units is dictated by the need to match the approximately exponential gap dependence of the magnetic force shown in Fig. 10. Assuming that all spring units provide the same force, each must bear $1 / 18$ of the total magnetic force. Each spring unit contains four springs. In order to tune the loading curve of the spring unit, two slightly different custom-designed [27] springs, shown in Fig. 11, are assembled into the spring unit as shown in Figs. 2 and 3.

A sorting process is used to determine which springs will be used together in one spring unit. Each spring is measured individually in the tuning and calibration system shown in Fig. 12. The load curve for an individual spring is typically not perfectly uniform, but has small (reproducible) bumps. A computerized sorting of the springs selects springs whose nonuniformities cancel one another for installation into the same spring unit.

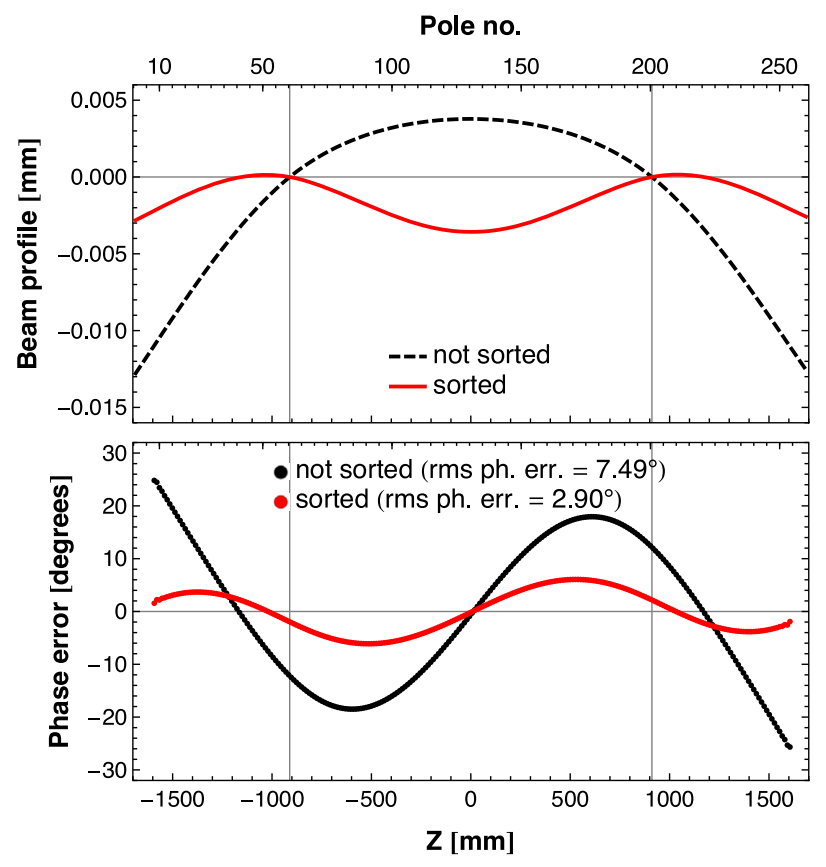

FIG. 9. Deformation of the strongback (top) and the resulting phase errors (bottom) vs distance along the undulator, for the set of random and systematic errors in spring unit strength of Fig. 8, and for the same set of errors after sorting. The vertical lines mark the actuator locations. 


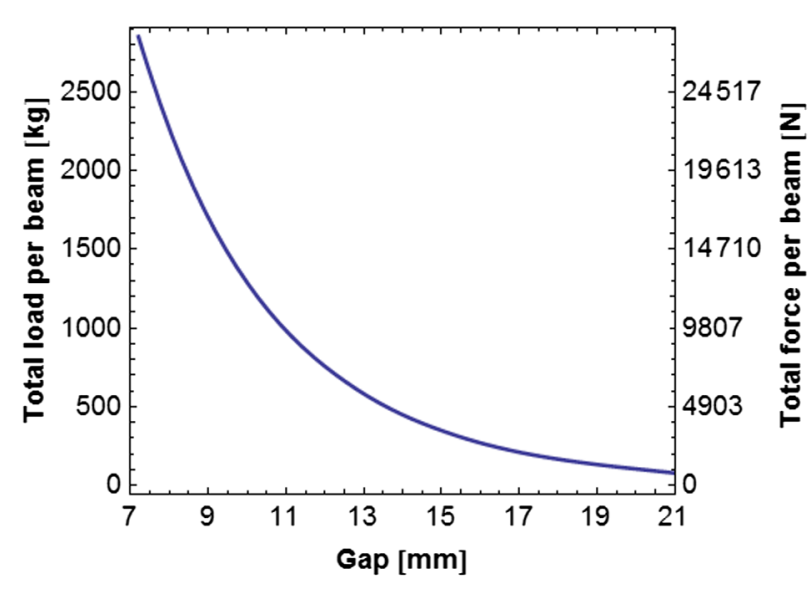

FIG. 10. Gap dependence of the magnetic force attracting the HGVPU strongbacks toward one another.

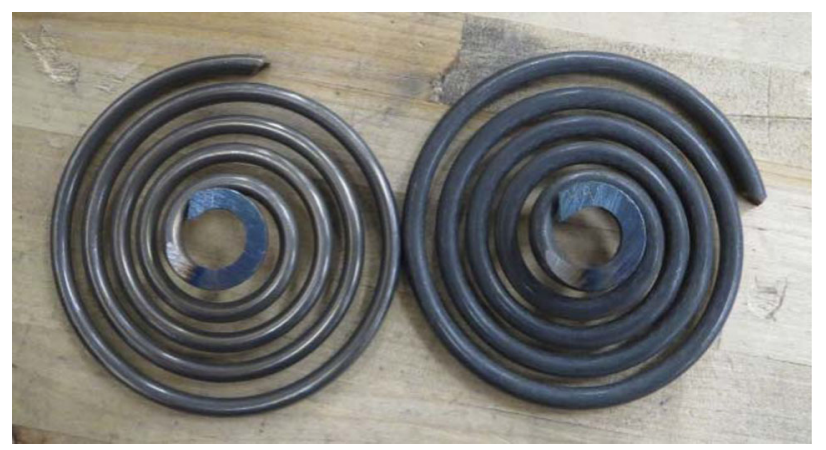

FIG. 11. The two types of springs used in the spring units. The weaker type is on the left and the stronger on the right.

The two types of springs have slightly different characteristic load curves. The spring calibration buttons shown in Fig. 3 are adjusted individually for each spring. Figure 13 illustrates how the two different load curves can be used to match the magnetic force profile. A pair of springs (one weak, one strong) has the engagement points of the springs adjusted differently in the two top panels of Fig. 13. In the left panel (labeled Tuning \#1), the strong spring has its engagement point shifted to larger gaps (i.e., the strong spring is strengthened) and the weak spring has its engagement point shifted to smaller gaps (i.e., the weak spring is weakened). The same two springs are adjusted in the opposite directions in the right panel (labeled Tuning \#2). The curves of total force from the two springs for the two tunings are shown in the bottom panel, along with the sum of the two untuned springs (the green curve). The alteration in the shape demonstrates how the force vs gap of the spring unit can be matched to the magnetic force vs gap.

The measured force curves for the springs are used to define the needed spring compression at minimum gap. The load curve is steep there, so the minimum-gap position corresponding to the desired force can be determined accurately. The software that selects the four springs for a spring unit also gives the minimum gap positions for the springs. The differences in those minimum gap positions are used to accurately set the relative heights of the spring calibration buttons during the assembly of the spring unit. For all but one or two of the spring units, no further adjustment in the individual spring calibration buttons was needed.

Once the spring units are assembled, the tuning and calibration system of Fig. 12 is also used for measuring the assembled spring units. The system allows positioning of the spring unit shaft to a precision of $0.1 \mu \mathrm{m}$ and, using the load cell, allows force measurements with $0.9-\mathrm{N}$ precision. The spring unit is secured in the calibration system in the same way it is fastened to the undulator girder. The shaft of the spring unit is directly connected to the motorized linear slide. In that way, the action of the spring unit in the undulator is fully duplicated. The calibration system, in an automated process, carries out multiple scans and records the loading curves.

The final result for the load curve corresponds well to the desired curve. The difference between the measured force curve and the desired curve is shown in Fig. 14 for the worst spring unit. Some residual bumps in the force curve can be seen, but the force still matches the desired value within $20 \mathrm{~N}(2 \mathrm{~kg})$ over the relevant gap range. This is

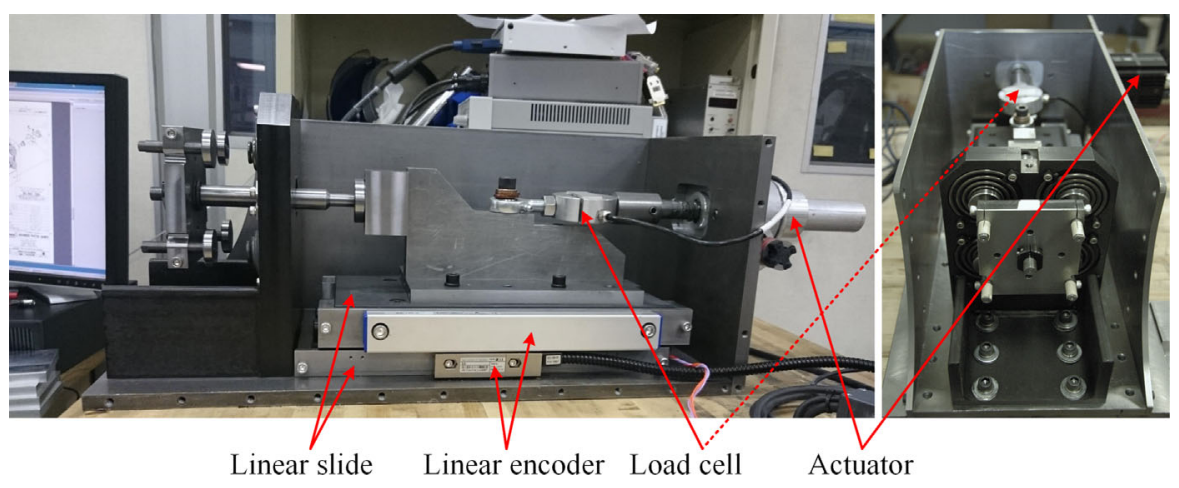

FIG. 12. Spring unit calibration system. The side panel and the actuator motor have been removed in the photo on the left and are in place in the photo on the right. 

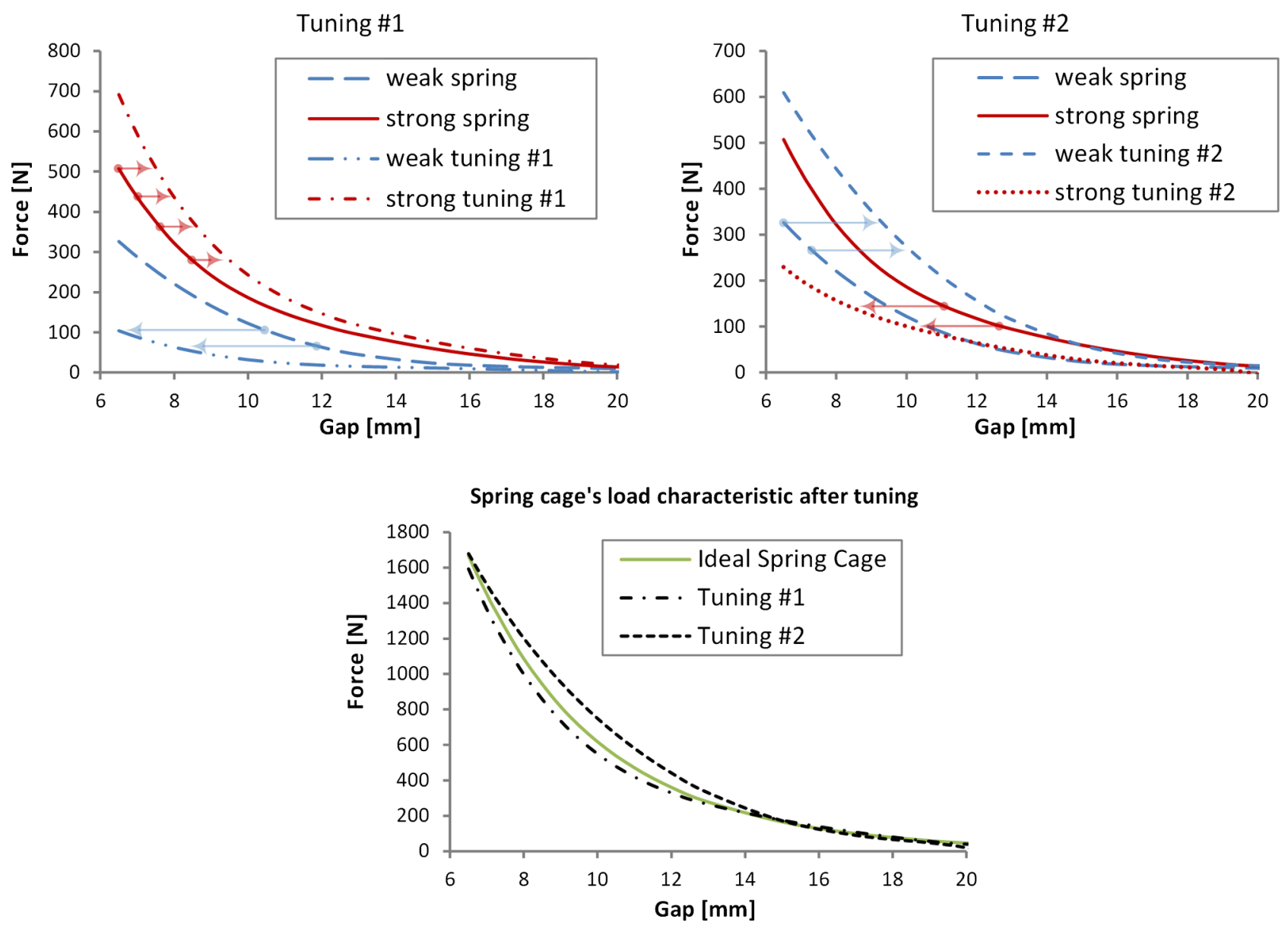

FIG. 13. An example of how the load characteristic of a spring unit can be tuned by adjusting the engagement point of the individual springs. A pair of springs_ - one strong, one weak_-has its engagement points shifted one way in "Tuning \#1" and the other way in "Tuning \#2." The resulting total force from the pair of springs in both cases is shown in the bottom panel, along with the total force from the untuned pair of springs.

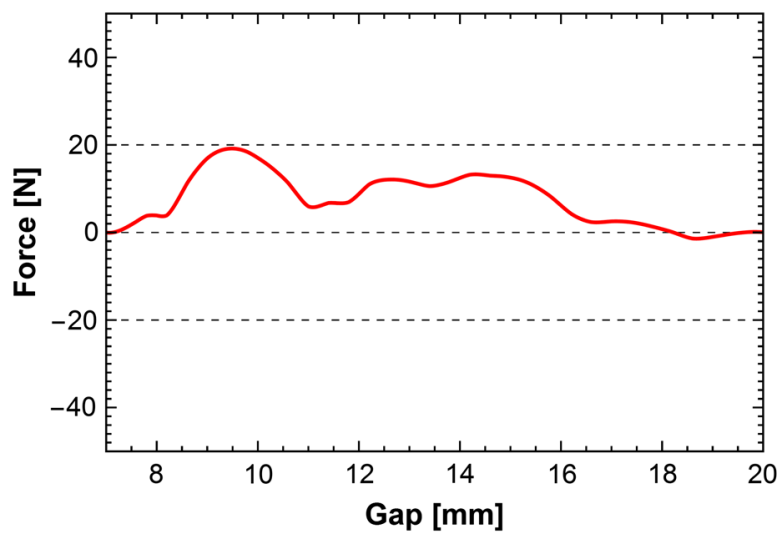

FIG. 14. The difference between the force of the worst of the spring cages and 1/18 of the magnetic attractive force of the undulator jaws is shown as a function of gap. Some bumpiness arising from imperfectly uniform springs is visible, but the force of the tuned spring unit matches the desired force within $\pm 2 \mathrm{~kg}$ over the relevant gap range, as shown by the dashed lines. smaller than the $5 \mathrm{~kg}$ random errors assumed for the calculations of Figs. 8 and 9.

Once assembled, each spring unit was calibrated and set to the position that would correspond to an undulator gap of $30 \mathrm{~mm}$. A clamp was applied that would lock the compression shaft of the spring unit in place so that when the spring units were installed on the HGVPU they all had the gap set to $30 \mathrm{~mm}$. There is almost no magnetic force at this gap, so the ID gap separation mechanism can be operated without magnetic force compensation. The pole gap was set to $30 \mathrm{~mm}$ using a gauge block. During installation the gap was monitored to ensure that the position of the spring units was correct within $\pm 5 \mu \mathrm{m}$.

\section{HGVPU MECHANICAL TUNING AND PERFORMANCE}

The gap straightness and uniformity requirements for the HGVPU are very demanding, so it is essential to have good methods provided to conveniently make precise 
adjustments. Adjustment of the mechanical gap on APS undulators has traditionally been done by inserting metal shims of various thicknesses between the magnet/pole holder and the aluminum strongback. This is a simple and reliable technique, but it has several drawbacks. The choice of shim thicknesses is limited so the uniformity of pole tip heights can only be adjusted with a resolution of $12.5 \mu \mathrm{m}$. Also, it requires loosening the magnet/pole holder mounting bolts, inserting or removing the shims between the strongback and the magnet/pole holder, and retightening the bolts before remeasuring. Since the process requires several iterations, it can be time consuming.

Therefore, a new gap mechanical alignment technique was implemented in the HGVPU. Belleville washers were added between the magnet/pole holder and the strongback (see Fig. 3). They are installed on the magnet/pole holder mounting bolts and fit into special sockets. During installation of a magnet/pole holder on the strongback, the mounting bolts are tightened until there is a $150-\mu \mathrm{m}$ gap between the magnet/pole holder and the strongback. This preloads the Belleville washers by approximately $4450 \mathrm{~N}$, an order of magnitude larger than the magnetic force generated at minimum gap. Thus the compression of the washers is not affected by gap-related changes in the magnetic force. Instead, the Belleville washers set and hold the spacing between the magnet/pole holder and the strongback. That spacing can be adjusted in the vicinity of the bolt location by slightly tightening or loosening the bolts, thereby allowing a continuous and rapid adjustment of the local gap. The adjustment range is $\pm 150 \mu \mathrm{m}$, enough to compensate for strongback, magnet/pole holder, and pole height imperfections.

The mechanical tuning of the HGVPU was performed at a gap of $8 \mathrm{~mm}$ because good mechanical tuning at small gap is most critical to the final overall performance of the undulator. As shown in Fig. 7, the pole heights must reproduce after gap cycling to $< \pm 7 \mu \mathrm{m}$ at the smallest gap. The requirement can be looser at larger gaps.
Measurements of the gap profile were performed with the Capacitec gap measurement system shown in Fig. 15. The system consists of two Capacitec HPS-1 1 4G-A-200-FX sensors mounted back to back with a distance adjuster. Each sensor must be close to its plane of pole tips $(<1 \mathrm{~mm})$; the distance adjuster holds the sensors appropriately for the gap being measured. The sensor dimensions are $1.0 \mathrm{~mm}$ horizontal by $4.0 \mathrm{~mm}$ vertical. Each sensor is calibrated by the vendor to an accuracy of $0.1 \mu \mathrm{m}$. Having two sensors allows the profile of each strongback to be measured individually as well as measuring the profile of the gap.

The measured profiles of the gap and of the individual undulator jaws are shown in Fig. 16 for a few gaps after mechanical tuning. The straightness of the strongbacks and overall gap remains within $\pm 10 \mu$ m over the entire gap range including gap $7.2 \mathrm{~mm}$, which meets the LCLS-II requirement.

The mechanical performance of the HGVPU was extensively studied because of the importance of accurate gap reproducibility for the LCLS-II undulators. Three separate sets of tests were performed, two of which were aimed at testing the performance of the spring units. In the first test, one of the HGVPU spring units was put through 30,000 compression cycles, going between the completely relaxed position and the fully compressed position that corresponds to an ID gap of $7.2 \mathrm{~mm}$. The calibration system of Fig. 12 was used for the test.

The results of the test are shown in Fig. 17. The left panel shows the force at maximum compression; the drift in this force is less than $1.4 \mathrm{~N}$. The right panel shows the force from the spring unit vs the gap (or, equivalently, the encoder position in the calibration system). A drift of $1.4 \mathrm{~N}$ is approximately $0.1 \%$ of the force generated by the spring unit. The $\sigma$ deviation of the force for different compression positions is below $1.2 \mathrm{~N}$. The jumps seen near maximum spring compression are due to some noise in the recording of the encoder position during several cycles and are not connected with any spring unit degradation.
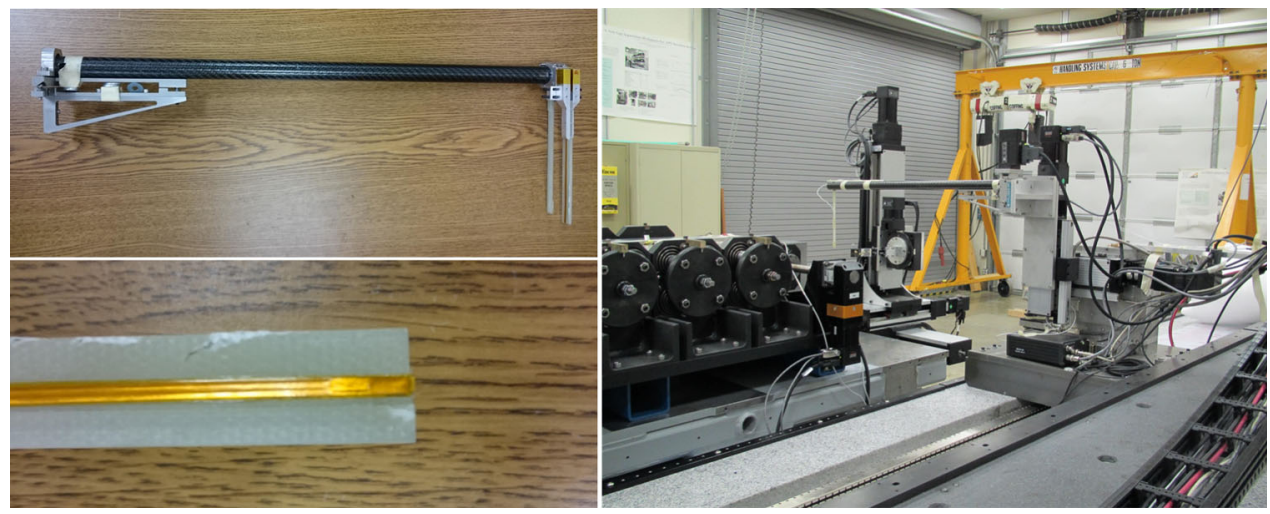

FIG. 15. The lower left panel shows one of the Capacitec sensors used to measure the gap profile. The Capacitec holder is shown in the top left panel. On the right, the sensors are shown installed and ready for use in the APS magnetic measurement facility. 

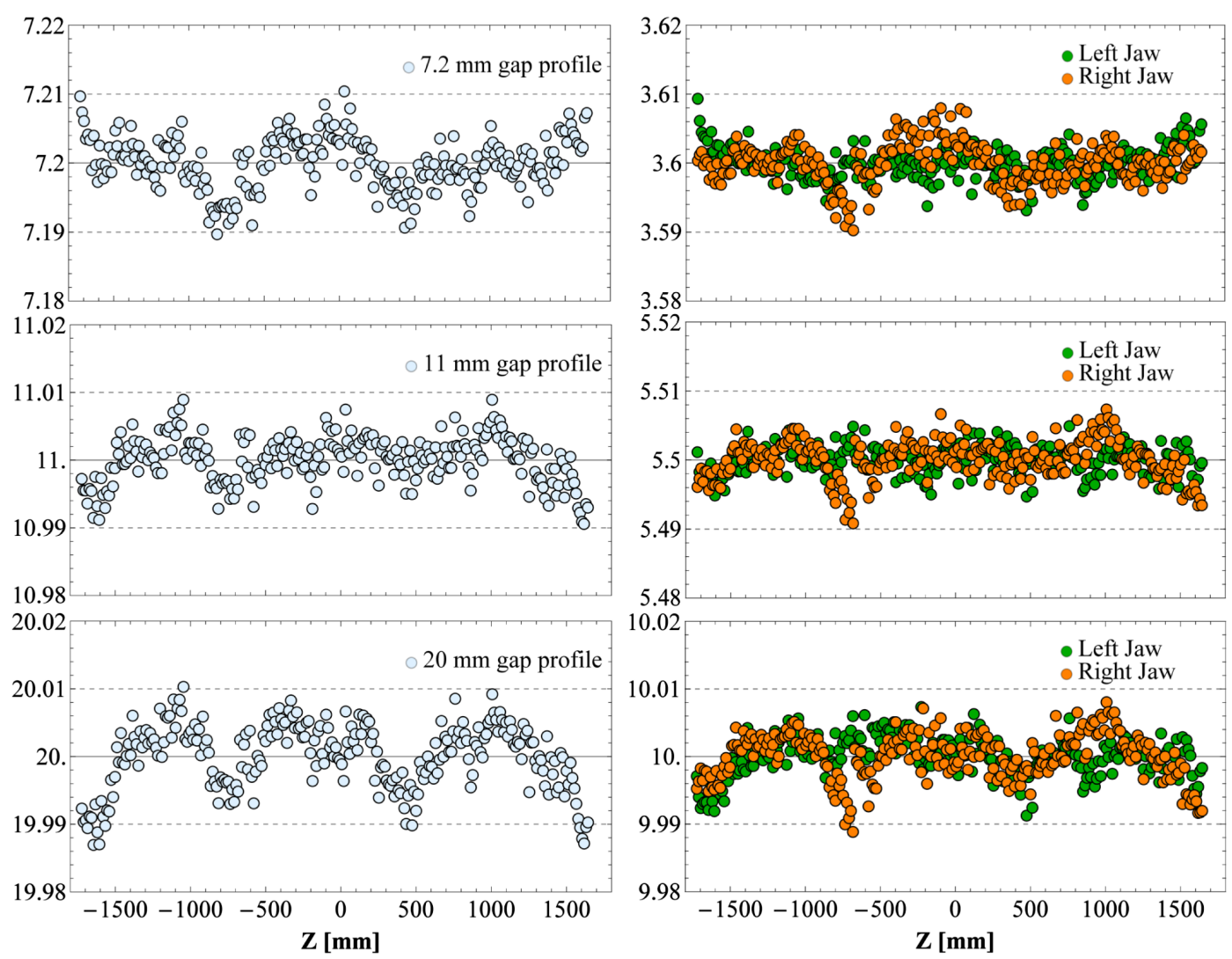

FIG. 16. Measured total gap in $\mathrm{mm}$ (left) and distance in $\mathrm{mm}$ between the gap center line and the pole tips on the left and right undulator jaws (right) vs z for several values of the gap. The horizontal dashed lines show that the mechanical tolerance of $\pm 10 \mu \mathrm{m}$ straightness has been met.

In the second test, the same spring unit was held at the maximum compressed position for three months $(\approx 2200$ hours). No degradation in performance was detected. These two tests show that the reliability and reproducibility of the spring units are very good and that they meet the lifetime performance requirements for LCLSII undulators.

The third test was to demonstrate the reproducibility of the gap by measuring the HGVPU magnetic performance.
The LCLS-II undulator must return to the same K parameter at any given gap to within $\Delta \mathrm{K} / \mathrm{K}<2.3 \times 10^{-4}$. The undulator gap was cycled more than 2000 times, and Hall probe scans were carried out after every 50 cycles. The effective $\mathrm{K}$ at a gap of $7.2 \mathrm{~mm}$ is shown as a function of the cycle count in Fig. 18. The test shows no evidence of long term drift or degradation in the effective field. The standard deviation of the measured data is 0.36 Gauss, as compared to the requirement of \pm 2.3 Gauss.
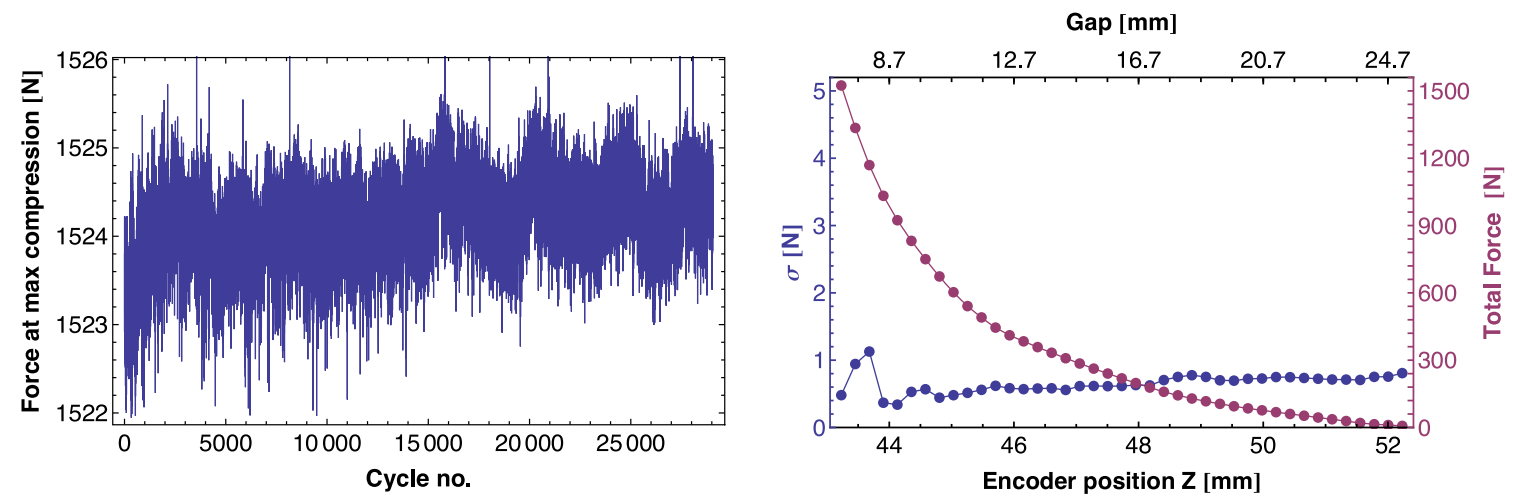

FIG. 17. Spring unit force at maximum compression (left), recorded over 30000 cycles. The force from the spring unit and the $\sigma$ deviation are shown at right vs gap. 


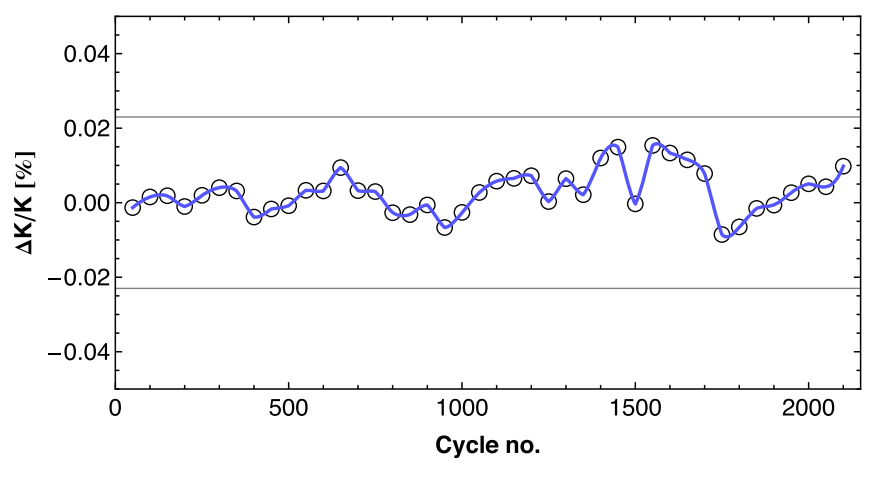

FIG. 18. Relative $\mathrm{K}_{\mathrm{eff}}$ change at a gap of $7.2 \mathrm{~mm}$, measured after every 50 cycles of the gap during a total of more than 2000 cycles. The requirement of $\pm 0.023 \%$ is indicated by the horizontal lines.

\section{ID MAGNETIC PERFORMANCE}

The final step of the HGVPU tuning process is the magnetic tuning. It includes tuning of the straightness of the trajectories in both horizontal and vertical directions, bringing the rms phase error to 4.0 degrees or lower for all operational gaps, and correcting the first and second field integrals $\left(\mathrm{J} 1_{x, y}\right.$ and $\left.\mathrm{J} 2_{x, y}\right)$ for all operational gaps. Multipole moments were also corrected.

The magnetic tuning used at APS is magnetic shimming. If the mechanical tuning has been done well and the magnet
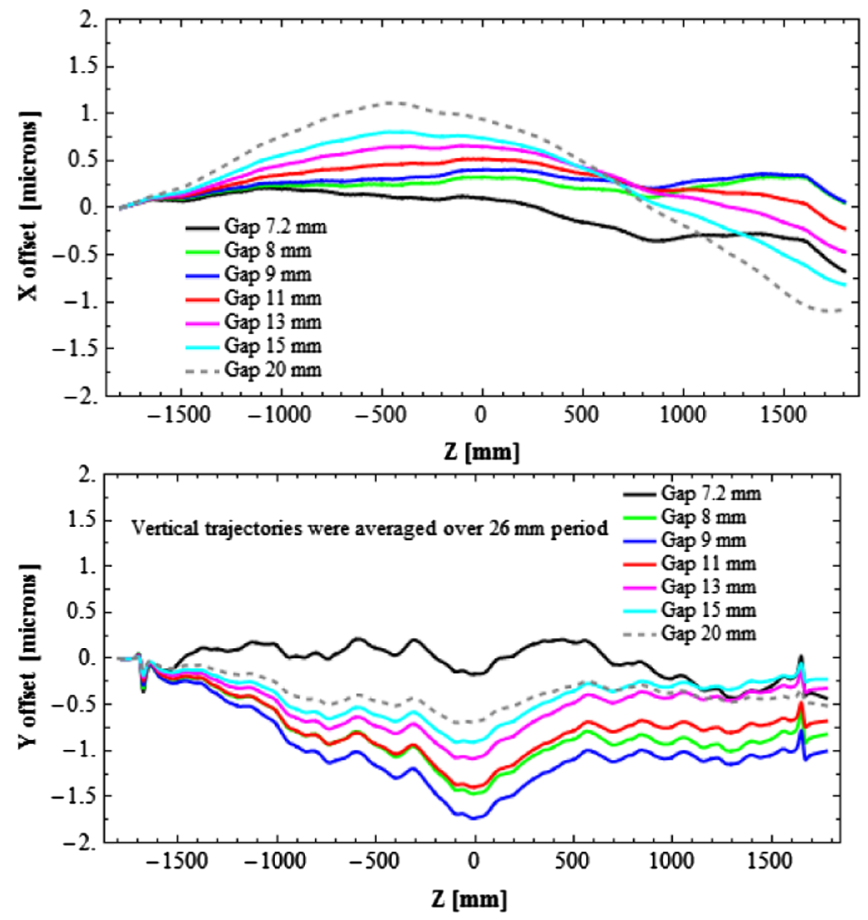

FIG. 20. Horizontal (top) and vertical (bottom) trajectories calculated from the magnetic field profiles at several gaps after magnetic tuning. Electron energy is taken to be $14 \mathrm{GeV}$. The limits on the graph are the $\pm 2 \mu \mathrm{m}$ straightness requirement.

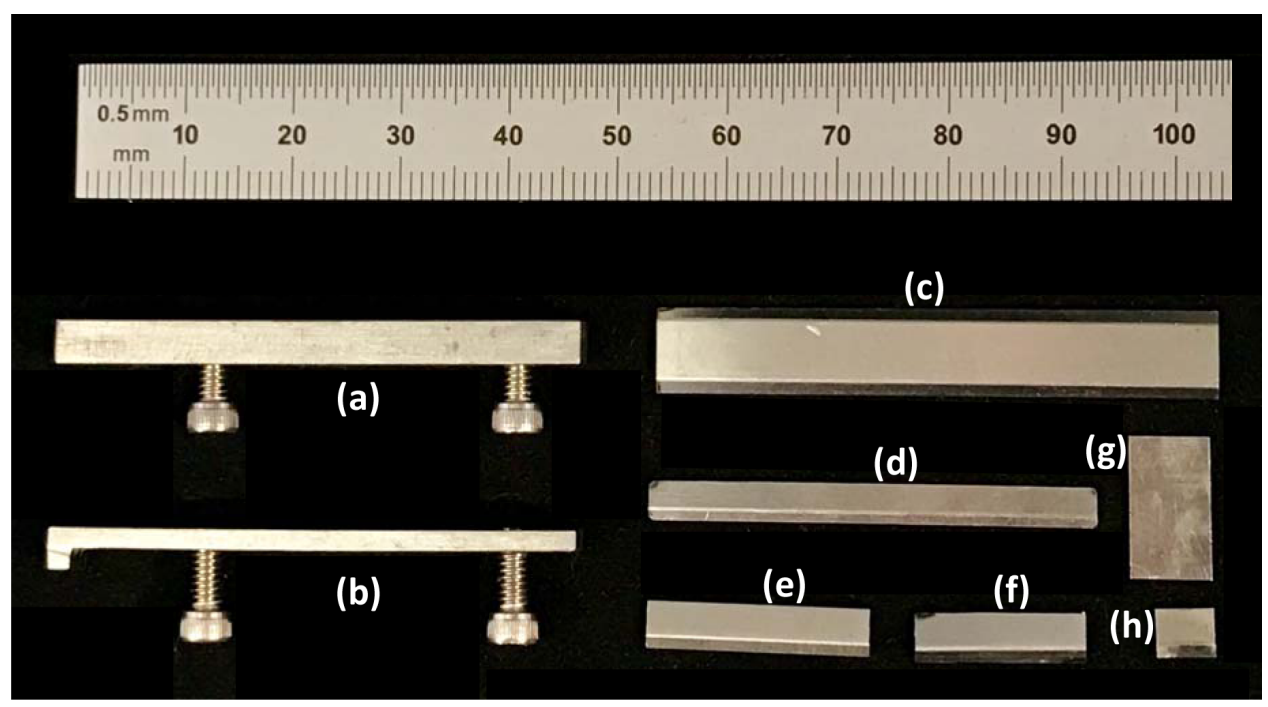

FIG. 19. Photograph of the types of magnetic shims used in tuning the HGVPU. (a) and (b) are side shims, placed on the sides of the pole for strong trajectory adjustments. Magnetic forces hold them in place; the screws are used to adjust their distance from the side of the pole. (c) is a phase shim, placed to cover the entire gap side of the magnet (the tip of the magnet is recessed from the pole tips by $0.5 \mathrm{~mm}$ so there is space for a 0.1- or 0.2-mm-thick shim). The bent edges shape the shim to conform to the magnet chamfers. (g) is a flat shim, placed to span from the center in $\mathrm{z}$ of one magnet to the center in $\mathrm{z}$ of the next magnet, immediately adjacent to the outside edge of the pole so the minimum gap is unaffected. It is a weaker version of the side shim. (d), (e), (f), and (h) are trajectory shims of various lengths in y that cover only half of the z-distance of the magnet face, with the bend to conform to the magnet chamfer. They may or may not be centered on the magnet in y depending on the correction needed. Different lengths and placements allow for different trajectory and multipole corrections, as do asymmetric (only one side) placements of the side and flat shims. 
TABLE III. Measured rms phase errors and effective field for the LCLS-II prototype.

\begin{tabular}{lcc}
\hline \hline Gap, mm & RMS phase error, degrees & B(effective), gauss \\
\hline 7.2 & 3.34 & 10604 \\
8.0 & 2.96 & 9451 \\
9.0 & 3.33 & 8788 \\
11.0 & 3.09 & 6217 \\
13.0 & 2.62 & 4777 \\
15.0 & 2.50 & 3705 \\
20 & 1.44 & 1997 \\
\hline \hline
\end{tabular}

block strength uniformity is good, magnetic shimming can correct the remaining issues. An automated process for the tuning of the first and second field integrals has been developed and successfully applied to the tuning of the HGVPU. The algorithm operates quite effectively and made the correct choices of shims from the "tool box" of available shims shown in Fig. 19. The tuning process for the trajectory and phase errors is yet to be automated. Instead, it is based on a visual examination of the trajectory and placement of relevant shims where needed.

Straightness of the trajectory in both horizontal and vertical directions and rms phase errors are important parameters for free electron laser undulators. Requirements for the LCLS-II undulator include a trajectory straightness of $< \pm$ $2 \mu \mathrm{m}$ and rms phase errors of 4.0 degrees or less for all operational gaps.

Magnetic tuning of phase errors and of the trajectory begins at the minimum gap of $7.2 \mathrm{~mm}$. After tuning at minimum gap, the trajectory measurements were verified at several other operational gaps. The trajectory deviation was within $\pm 2.0 \mu \mathrm{m}$ and the rms phase errors were below 4.0 degrees for all operational gaps (Fig. 20 and Table III). In Fig. 20, a steering corrector has been assumed at the upstream end of the device. In a real scenario, the corrector would be placed farther upstream.

The effective field is greater than $1.06 \mathrm{~T}$ at a gap of $7.2 \mathrm{~mm}$, exceeding the LCLS-II requirement of $1.01 \mathrm{~T}$. The trajectories and phase errors were measured using a Senis model H3 A-YZP02 F-B02T5K0 K Hall probe. The APS

TABLE IV. LCLS-II field integrals.

\begin{tabular}{lcccc}
\hline \hline Gap, mm & $\begin{array}{c}\mathrm{J} 1 \mathrm{x} \\
(\mathrm{G}-\mathrm{cm})\end{array}$ & $\begin{array}{c}\mathrm{J} 2 \mathrm{x} \\
\left(\mathrm{kG}-\mathrm{cm}^{2}\right)\end{array}$ & $\begin{array}{c}\mathrm{J} 1 \mathrm{y} \\
(\mathrm{G}-\mathrm{cm})\end{array}$ & $\begin{array}{c}\mathrm{J} 2 \mathrm{y} \\
\left(\mathrm{kG}-\mathrm{cm}^{2}\right)\end{array}$ \\
\hline 7.2 & 15 & -0.9 & 36 & 2.3 \\
8.0 & -37 & -4.6 & 26 & 0.2 \\
9.0 & -33 & 0.2 & 22 & -0.4 \\
11.0 & -17 & 5.5 & 22 & 0.4 \\
13.0 & -2 & 3.7 & 20 & 2.5 \\
15.0 & 10 & 5.5 & 13 & 4.1 \\
20 & 35 & 8.1 & -25 & 4.7 \\
Requirement & \pm 40 & \pm 15 & \pm 40 & \pm 15 \\
\hline \hline
\end{tabular}

TABLE V. LCLS-II integrated multipole moments.

\begin{tabular}{lcccccc}
\hline \hline & $\mathrm{a} 1$ & $\mathrm{a} 2$ & $\mathrm{a} 3$ & $\mathrm{~b} 1$ & $\mathrm{~b} 2$ & $\mathrm{~b} 3$ \\
Gap & $(\mathrm{G})$ & $(\mathrm{G} / \mathrm{cm})$ & $\left(\mathrm{G} / \mathrm{cm}^{2}\right)$ & $(\mathrm{G})$ & $(\mathrm{G} / \mathrm{cm})$ & $\mathrm{G} / \mathrm{cm}^{2}$ \\
\hline 7.2 & -20 & -129 & 270 & -26 & 83 & -171 \\
8.0 & -36 & -89 & 199 & -1 & 48 & -127 \\
9.0 & -41 & -71 & 12 & -1 & 42 & -151 \\
11.0 & -15 & 12 & -82 & 6 & -17 & -32 \\
13.0 & 10 & 12 & -71 & 6 & -4 & -32 \\
15.0 & 24 & 25 & 39 & 59 & -16 & -50 \\
20 & 68 & 23 & -39 & 26 & 25 & -18 \\
Requirement & \pm 100 & \pm 200 & \pm 400 & \pm 100 & \pm 200 & \pm 400 \\
\hline \hline
\end{tabular}

Hall probe measurements were taken on the fly with a longitudinal speed along the device of $150 \mathrm{~mm} / \mathrm{s}$.

Magnetic shims (see Fig. 19) are used to meet the requirements for the first $(\mathrm{J} 1 \mathrm{x}, \mathrm{y})$ and second $(\mathrm{J} 2 \mathrm{x}, \mathrm{y})$ field integrals as well as the skew and normal quadrupoles (a1, b1), sextupoles (a2, b2), and octupoles (a3, b3). Table IV shows the first and second integral tuning results. Table $\mathrm{V}$ shows the multipole tuning results.

\section{CONCLUSION}

A 3.4-meter long HGVPU with dynamic compensation of the magnetic forces has been successfully designed, built, tuned, and tested at the APS. The design is "gravity neutral" and uses a novel spring system for the dynamic compensation of the ID magnetic forces. The device delivers vertically polarized radiation. The performance measurement results show that all LCLS-II requirements have been met or exceeded. As a result, the HGVPU has been included in the baseline of the LCLS-II project for the hard x-ray undulator line.

\section{ACKNOWLEDGMENTS}

The authors wish to thank Mark Overmyer of Rockford Spring Company for his assistance with and customized design of the variable-response springs used in this project. This work was supported by U.S. Department of Energy, Office of Basic Energy Sciences, under Contract No. DEAC02-06CH11357.

[1] J. A. Clarke, The Science and Technology of Undulators and Wigglers (Oxford University Press, New York, 2004).

[2] E. Levichev and N. Vinokurov, Undulators and other insertion devices, Rev. Accel. Sci. Technol. 03, 203 (2010).

[3] D. Fritz, SLAC Report No. SLAC-R-1048, 2014.

[4] Technical Design Report No. DESY 2006-097, 2007.

[5] D. E. Kim, H. S. Han, Y.-G. Jung, H.-G. Lee, W. W. Lee, K.-H. Park, H. S. Suh, and J. Pflueger, Status of the PAL-XFEL undulator system, in Proceedings of the 3rd International Particle Accelerator Conference, New Orleans, Louisiana, USA, 2012 (IEEE, Piscataway, 
2012), p. 3509; D. E. Kim, H. S. Han, Y.-G. Jung, H.-G. Lee, W.-W. Lee, K.-H. Park, H.-S. Suh, S.-B. Lee, I.-S. Ko, and M.-H. Cho, First results of the PAL-XFEL prototype undulator measurements, in Proceedings of the 4th International Particle Accelerator Conference, IPAC2013, Shanghai, China, 2013 (JACoW, Shanghai, China, 2013), p. 3561.

[6] E. Wallén et al., Status of the LCLS-II undulators, AIP Conf. Proc. 1741, 020025 (2016).

[7] T. Tanaka, S. Goto, T. Hara, T. Hatsui, H. Ohashi, K. Togawa, M. Yabashi, and H. Tanaka, Undulator commissioning by characterization of radiation in $\mathrm{X}$-ray free electron lasers, Phys. Rev. ST Accel. Beams 15, 110701 (2012).

[8] T. Schmidt, P. Böhler, M. Brügger, M. Calvi, S. Danner, P. Huber, A. Imhof, H. Jöhri, A. Keller, M. Locher, T. Stapf, and J. Wickstroem, SwissFEL U15 prototype design and first results, in Proceedings of FEL2012, Nara, Japan, p. 666; SwissFEL conceptual design report, (Paul Scherrer Institut, Switzerland, 2012), pp. 91-102.

[9] D. La Civita, R. Bracco, B. Diviacco, G. Tomasin, and D. Zangrando, FERMI@Elettra undulator frame study, in Proceedings of the 11th European Particle Accelerator Conference, Genoa, 2008 (EPS-AG, Genoa, Italy, 2008), p. 2276; Conceptual design report, FERMI@Elettra Project, 2007, pp. 223-266.

[10] R. Carr, SLAC Report No. SLAC-PUB-9594, 2002.

[11] R. Kinjo, T. Tanaka, T. Seike, A. Kagamihata, and S. Yamamoto, Development of a magnet system to cancel the attractive force toward structural reform of undulators, in Proceedings of FEL2014, Basel, Switzerland, p. 75 .

[12] R. Kinjo and T. Tanaka, Phase combination for selfcancellation of magnetic force in undulators, Phys. Rev. ST Accel. Beams 17, 122401 (2014).

[13] O. Marcouillé, P. Brunelle, O. Chubar, M.-E. Couprie, J.-M. Filhol, C. Herbeaux, J.-L. Marlats, A. Mary, and K. Tavakoli, An in vacuum wiggler WSV50 for producing hard $\mathrm{x}$ rays at SOLEIL, in Proceedings of the 11th
European Particle Accelerator Conference, Genoa, 2008 (Ref. [9]), p. 2288.

[14] D. Waterman, Support structures for planar insertion devices, U.S. Patent No. 7956557, 2011.

[15] E. Gluskin, E. Trakhtenberg, and J. Z. Xu, Undulator with dynamic compensation of magnetic forces, U.S. Patent No. 9355767, 31 May 2016.

[16] N. Strelnikov, E. Trakhtenberg, I. Vasserman, J. Xu, and E. Gluskin, Vertically polarizing undulator with the dynamic compensation of magnetic forces for the next generation of light sources, Rev. Sci. Instrum. 85, 113303 (2014).

[17] Opera Simulation Software, Cobham, Oxfordshire, UK, http://www.operafea.com.

[18] R. P. Walker, Phase errors and their effect on undulator radiation properties, Phys. Rev. ST Accel. Beams 16, 010704 (2013).

[19] Y. Li, B. Ketenoglu, and J. Pflueger, Girder deformation related phase errors on the undulators for the European X-ray free electron laser, Phys. Rev. ST Accel. Beams 18, 060704 (2015).

[20] COMSOL MULTIPHYSICS ${ }^{\circledR}$ v. 5.2. http:www.comsol.com. COMSOL AB, Stockholm, Sweden.

[21] L. D. Landau and E. M. Lifshitz, Theory of Elasticity (Pergamon, New York, 1970), Sec. 17-20.

[22] R. R. Craig, Mechanics of Materials, 2nd ed. (Wiley, New York, 1999).

[23] P. Elleaume, O. Chubar, and J. Chavanne, Computing 3D magnetic fields from insertion devices, in Proceedings of the Particle Accelerator Conference, Vancouver, BC, Canada, 1997 (IEEE, New York, 1997), p. 3509.

[24] RADIA, a 3D magnetostatics computer code, http:www.esrf .eu/Accelerators/Groups/InsertionDevices/Software/Radia.

[25] L. D. Landau and E. M. Lifshitz, The Classical Theory of Fields (Pergamon, New York, 1971), Sec. 33.

[26] J. D. Jackson, Classical Electrodynamics 3rd ed. (Wiley, New York, 1998).

[27] Rockford Spring Company, 3801 South Central Ave., Rockford, IL 61102, http://www.rockfordspring.com. 